\title{
Species Identification of Non-tuberculous Mycobacteria (NTM) from Sputum Samples of TB Suspects in a Tertiary Care Centre from North India
}

\section{Rohini Sharma ${ }^{1}$, Binit Kumar Singh ${ }^{1}$, Pankaj Jorwal ${ }^{1 *}$, Indra Mani ${ }^{1}$, Vishwanath Upadhyay ${ }^{1}$, Manish Soneja ${ }^{1}$, George Sebastian ${ }^{3}$, Ranjani Ramachandran ${ }^{2}$, Prahlad Kumar ${ }^{3}$ and Naveet Wig ${ }^{1}$}

${ }^{1}$ Department of Medicine, All India Institute of Medical Sciences, New Delhi, India ${ }^{2}$ TB Lab Focal Point, World Health Organization New Delhi, India

${ }^{3}$ National Tuberculosis Institute, Bangalore, India of Medical Sciences, New Delhi, India.

Received: July 27, 2021

Published: August 21, 2021

(C) All rights are reserved by Pankaj Jorwal., et al.

\begin{abstract}
Purpose: Non-tuberculous Mycobacteria (NTM) infections are often misdiagnosed as tuberculosis due to the similar clinical and radiological presentations. NTM infections can be differentiated from tuberculosis only through species identification. Here we performed a species-level identification of NTM from sputum samples using ITS sequencing of the 16S-23S rRNA gene.

Materials and Methods: Twenty-one immunochromatographic assay negative isolates from smear-positive patients were collected and evaluated. The isolates were then subjected to mycolic acid extraction for HPLC, DNA isolation for line probe assay (LPA), $h s p 65$ and 16S-23S rRNA gene ITS sequencing.

Results: After evaluating all the diagnostic modalities for NTM, it was observed that the Mycobacterium intracellulare was the predominant slow-growing NTM (9/20, 45\%), while Mycobacterium abscessus (subsp. bolletii) was the dominant rapid grower (5/20, $25 \%$ ). One isolate, which could not be identified either by LPA or by HPLC, was detected as Nocardia cyriacigeorgica, by sequencing a non mycobacterial species predominantly found in pulmonary infection. The results of HPLC and LPA were 100\% concordant. One isolate was identified as M. abscessus by sequencing, but HPLC and LPA detected it as Mycobacterium chelonae.

Conclusion: NTM should be considered as the key pathogen in immunocompromised patients and in patients with manifestations similar to tuberculosis. In such cases, species identification is critical for initiation of appropriate therapy. The 16S-23S rRNA gene ITS sequencing method is a rapid and accurate technique for NTM species identification up to subspecies.
\end{abstract}

Keywords: Non-tuberculous Mycobacteria; Internal Transcribed Spacer Sequencing; Line Probe Assay; HPLC

\section{Key Messages}

Present study helps in understating the NTM infections which are often misdiagnosed and incorrectly treated as tuberculosis due to the similar clinical and radiological presentations. In this study, we performed species identification of NTM using ITS sequencing of the 16S-23S rRNA gene and compared the results to those obtained using HPLC and line probe assay. Our findings indicate that the use of ITS (16S-23S rDNA ITS sequences) region sequencing is a rapid and reliable way of NTM species identification.

\section{Introduction}

Non-tuberculous Mycobacteria (NTM) are being increasingly recognized as the cause of chronic pulmonary and extra pulmonary disease. Among pulmonary tuberculosis patients who do not respond to anti-tuberculosis therapy, a high index of suspicion should be maintained for NTM as an etiological agent, and appropriate testing should be conducted to isolate them. At present, more than 60 types of NTM species that are pathogenic to humans have been 
identified [1]. Their prevalence is especially high in immunocompromised hosts like PLHIV and organ transplant recipients, and early diagnosis and treatment is essential to prevent disease progression and further complications. The diseases caused by NTM present similar to tuberculosis, which often results in them being misdiagnosed as tuberculosis. Moreover, techniques for the identification of NTM are available only at select centres. Both these factors contribute to poor understanding of the epidemiology and disease burden of NTM. Traditional methods have relied on evaluation of the growth rate, pigment production, and colony morphology for preliminary identifications of NTM; however, rapid and accurate species identification is crucial to initiate appropriate therapy and to distinguish pathogenic species from contaminants [2].

The automated liquid culture system (MGIT 960) with commercial molecular techniques such as probe hybridization assay [GenoType Mycobacterium CM/AS (HAIN Life science, Germany)] can accurately identify species, yielding results within weeks [3]. High Performance Liquid Chromatography (HPLC) relies on an organism's mycolic acid (MA) content (fatty acids found in the cell walls of Mycobacteria) and is highly species specific; however, it is highly labour intensive and takes months to obtain the results [4]. Hence, there is a need for the development of a clinically useful and cost effective, rapid and accurate diagnostic test for species identification of NTM. The16S-23S rRNA gene internal transcribed spacer (ITS) sequencing technique is highly specific for identifying most Mycobacterial species [5]. Although, there are other genes such as $h s p 65, r p o B$ and $16 \mathrm{~s}$ rRNA genes were also used in various studies done worldwide [6-8]. In this observational study, we performed NTM species identification by ITS sequencing of 16S-23S rRNA gene region and compared the results of those obtained using probe hybridization assay [Geno Type Mycobacterium CM/AS [HAIN Lifescience, Germany)] and HPLC. We have also performed the $h s p 65$ gene sequencing as well to give more strength to NTM speciation experiments.

\section{Materials and Methods \\ Ethical statement}

The study was approved by institutional ethics committee and written informed consent was obtained from all the study participants.

\section{Subject recruitment and initial processing}

A total of 1619 sputum smear-positive samples [male; 1104 (68\%), female; 515 (32\%) with mean age of $35 \pm 14.5$ years] were received at Intermediate Reference Laboratory of the Department of Medicine at AIIMS, New Delhi between September 2013 and January 2016. All the patients were diagnosed with tuberculosis and were not responding to standard anti-tuberculosis therapy and were HIV negative. Of these, 21 were suspected as NTM based on an immunochromatographic assay (ICA)-negative in culturepositive isolates result (Figure 1). The cryopreserved cultures were revived and sub-cultured on liquid (BACTEC MGIT-960) and on Lowenstein Jensen (LJ solid culture) medium using standard operating procedures (SOP) of the National Tuberculosis Elimination Programme (formerly Revised National Tuberculosis Control programme) $[9,10]$. These were then observed for growth rate, pigment production, and colony morphology on LJ medium (Figure 2) and initially classified based on the Runyon classification criteria [11].

\section{HPLC and DNA sequencing}

The MA extraction for HPLC (Agilent HPLC instrument) and DNA isolation for line probe assay was performed for all the cul-

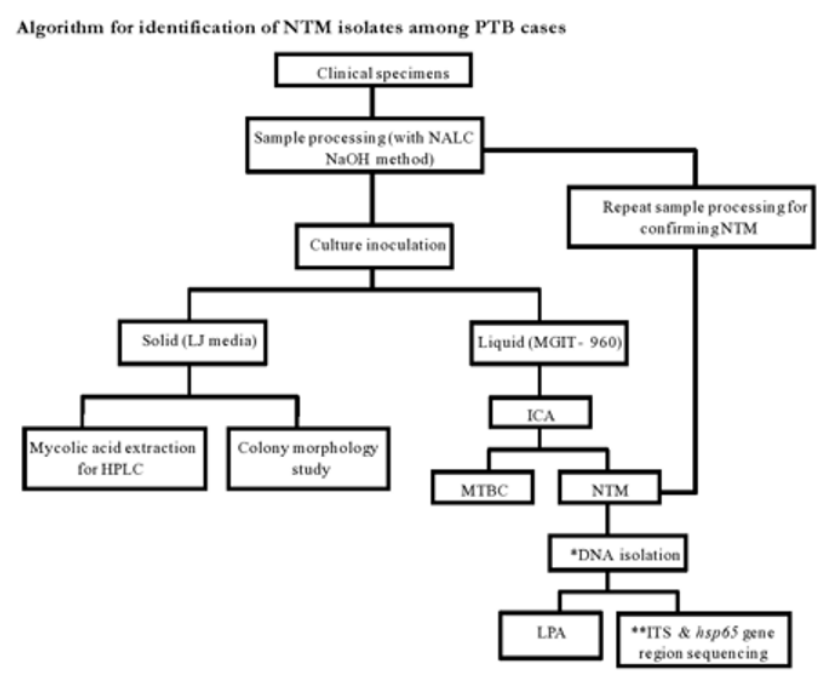

Figure 1: Schematic flow of species identification of NTM isolates.

Definition of abbreviations: MGIT-960: Mycobacterial Growth Indicator Tube 960; ICA: Immunochromatographic Assay; L-J: Lowenstein-Jensen Media; DNA*: Deoxyribose Nucleic Acid (DNA was used for LPA and ITS gene sequencing); DST: Drug Susceptibility Testing; CM/AS: Common Mycobacteria/Additional Species of Mycobacteria; LPA: Line Probe Assay; HPLC: High Performance Liquid Chromatography; AFB: Acid-fast Bacilli; MTBC: Mycobacterium tuberculosis Complex; Mtb: Mycobacterium tuberculosis; NTM: Non-tuberculous Mycobacteria; ITS** Internal Transcribed Spacer Region of Ribosomal Gene Amplification for Species Identification of NTM Isolate. 


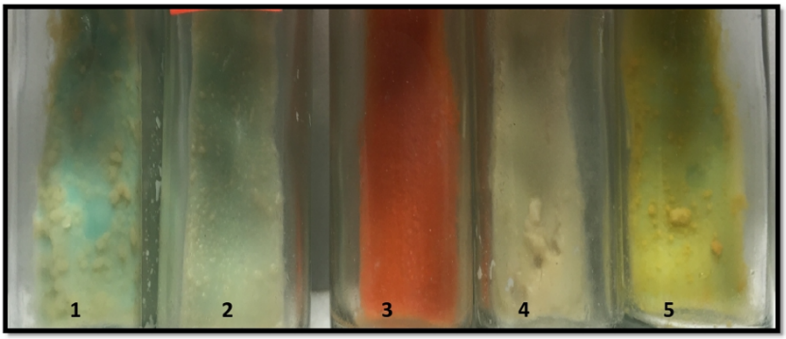

Figure 2: Identification of the NTM culture isolates on the basis of colony morphology on LJ media (solid culture). Lane 1: Mycobacterium tuberculosis (rough, buff and beige coloured colony), Lane 2: M. intracellurae (non pigmented shiney sticky coloney), Lane 3: M. gordonae pigment producer, Lane 4: M. simiae confluent growth and pigmented when exposed in light. Lane 5: $M$. absessus light yellow pigments producer.

tures as per the previous studies $[4,6,12]$. The isolated DNA was then subjected to DNA sequencing on ABI prism 3130xl genetic analyzer (Applied Biosystems and Hitachi, Ltd., Carlsbad, CA, USA). for hsp65 gene using primers Tb11(F): 5' -ACCAACGATGGTGTGTCCAT-3' and Tb12 (R): 5'-CTTGTCGAACGCATACCCT-3' and 16S-23S rRNA gene ITS region with the primers ITS(F) 5'-GAAGTCGTAACAAGGTAGCCG-3' and ITS(R) 3'- GACAGCTCCCCGAGGCTTATCGCA-5' (Supplementary Figure 1) [6].

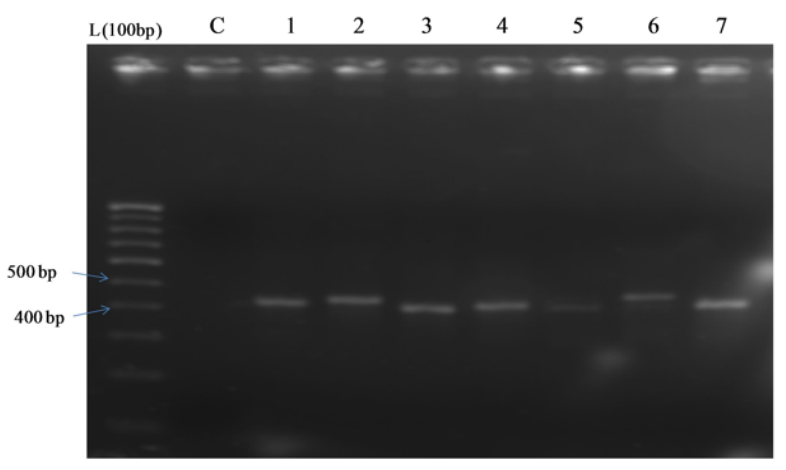

Supplementary Figure 1: Gel picture of ITS gene amplified product of NTM isolates from PTB specimens; L: ladder (100bp); C: DNA control; 1: M. intracellurae; 2: M. absessus; 3: M. gordonae; 4: M. simiae; 5: M. kansassi; 6: Nocardia species; 7: M. fortuitum.
Sequence analysis

The nucleotide sequence of the ITS region and $h s p 65$ gene of the NTM isolates were analysed using a Basic Local Alignment Search Tool (BLAST) (http://www.ncbi.nlm.nih.gov) (Thomas Hall, Raleigh, NC, USA). Conservative and polymorphic ITS and hsp65 sequences of the NTM isolates were presented in a multi-alignment of the ITS and hsp65 regions using CUSTAL-W 2.0 software (http:// genome.kribb.re.kr). The obtained nucleotide sequences were submitted to NCBI GenBank and an accession number for ITS sequence was generated for each identified isolate [Supplementary Figure 2 (1-3)].

\section{Results}

Of the 1619 smear-positive sputum specimen received, 21 ICAnegative isolates were tested using the GenoType ${ }^{\circledR}$ Common $M y$ cobacteria (CM) kit, HPLC and sequencing simultaneously. Among these, nine (45\%) were identified as Mycobacterium intracellulare, three (15\%) as Mycobacterium gordonae, five (25\%) as Mycobac-

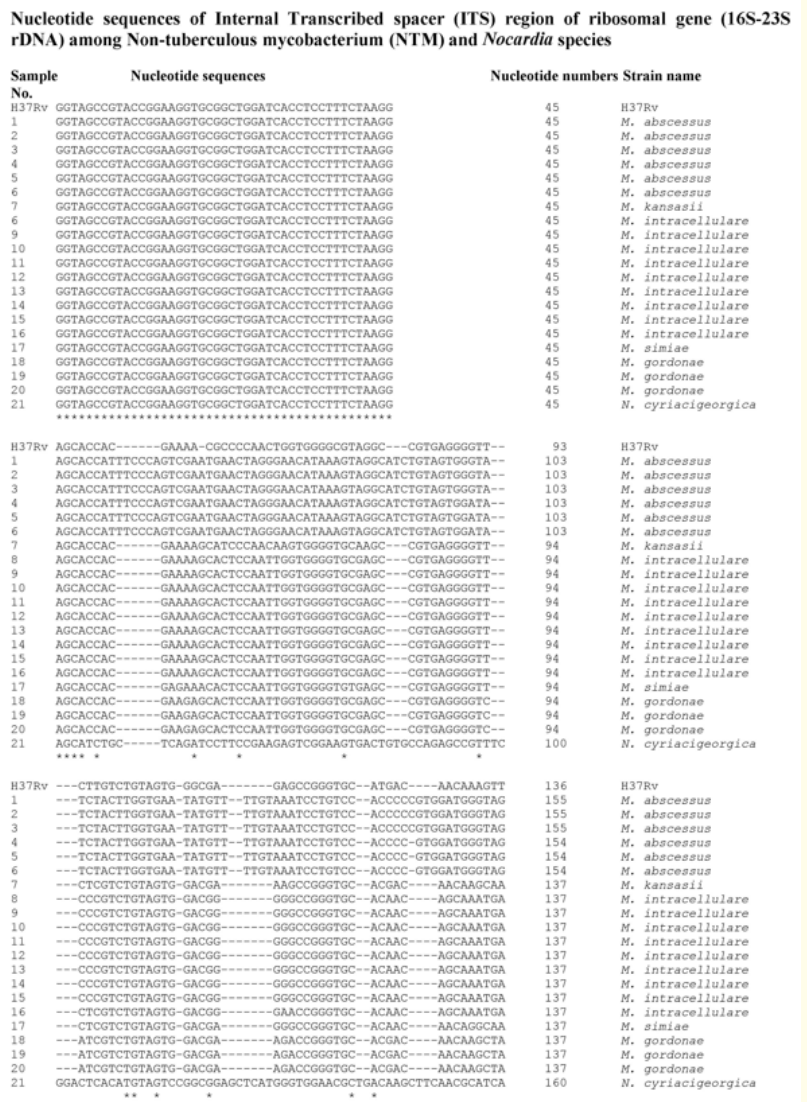



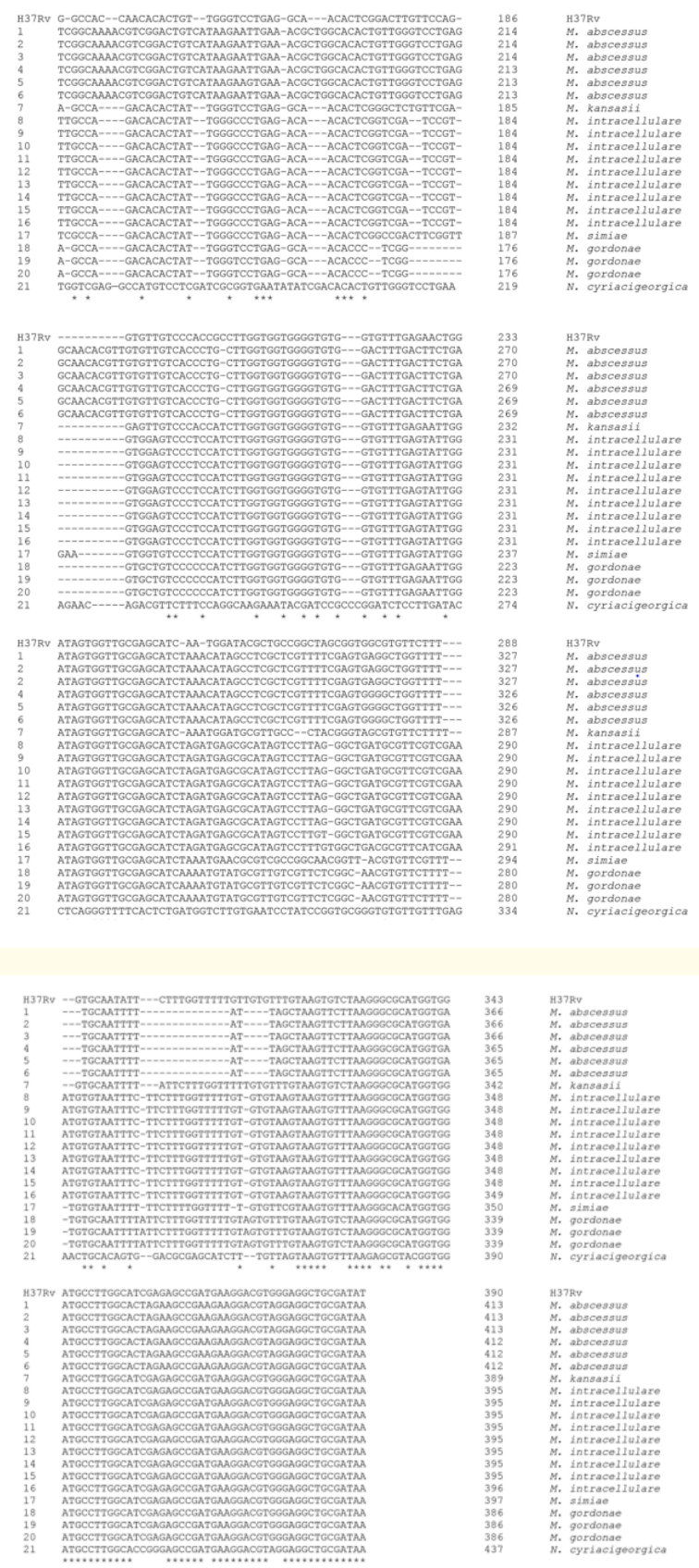

Supplementary Figure 2: Alignment of 16S-23S rDNA ITS sequences (sample number 1" "-20 $^{0 \text { " }}$ NTM species and $21^{*}$ Nocardiaspp.)

Supplementary Figure 2: Alignment of 16S-23S rDNA ITS sequences (sample number $1^{\text {st }}-20^{\text {th }}$ NTM species and $21^{\text {st }}$ Nocardiaspp.).

terium abscessus, and one (5\%) as Mycobacterium chelonae. Two unidentified isolates were tested using the GenoType ${ }^{\circledR}$ Additional species of Mycobacteria (AS) kit; one (5\%) was identified as Mycobacterium simiae and the other was found to not be a NTM upon analysis using the GenoType ${ }^{\circledR}$ AS kit. Howerver, one isolate were found to be invalid at the end of hybridization of GenoType ${ }^{\circledR}$, were found as Mycobacterium kansasii $(1 ; 5 \%)$ in HPLC and sequencing simultaneously (Figure $3 \mathrm{a}$ and $3 \mathrm{~b}$ ).

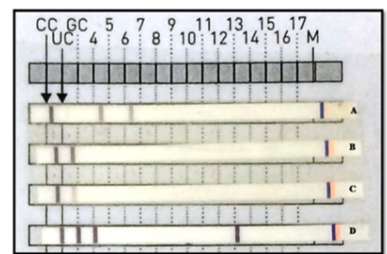

(a)

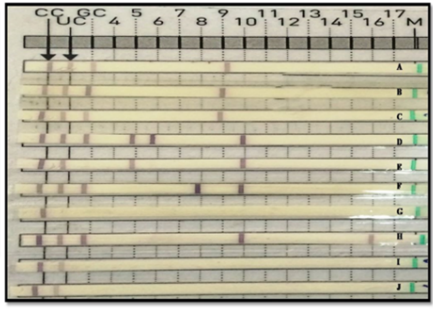

(b)
Figure 3

a. DNA strips of GenoType Mycobacterium AS kit.

A: showing $M$. simiae NTM species identified by using GenoTypeR Mycobacterium AS kit; B: Negative control; C: Master mix control, D: M. tuberculosis H37RV positive control.

b. DNA strips of GenoType Mycobacterium CM kit.

A: M. intracellulare; B: M. intracellurae; C: M. intracellurae; D: M. absessus; E: M. chelonae; F: M. gordonae; G: GC-Negative; H: (H37RV) I: Negative control; J: Master mix control.

The prepared MA extract was subjected to HPLC (Agilent HPLC instrument) and analysis was performed using a UV detector. The obtained HPLC peaks of the NTM isolates were separated into clusters (single, double, triple, and multiple clusters) based on high and low molecular weight. Each cluster group was then identified by the number of peaks, retention times, and relative peaks heights. Of the 21 ICA-negative isolates, 20 were separated using HPLC into clusters and peaks. Among the single clusters, three (15\%) were identified as M. gordonae, one (5\%) as M. kansasii, previously this isolate was observed as invalid in GenoType ${ }^{\circledR}$ and one was a $\mathrm{H}_{37 \mathrm{R}_{\mathrm{v}}}$ as control. The double cluster included five (25\%) M. abscessus isolates, one (5\%) M. chelonae isolate, and 9(45\%) M. intracellulare isolates; the triple clusters included only one (5\%) M. simiae isolate (Figure $4 \mathrm{a}$ and $4 \mathrm{~b}$ ).

The results of ITS and $h s p 65$ genes based sequencing were identical in speciation of NTM isolates and mostly consistent with those of LPA and HPLC. One discordant result was obtained by ITS and hsp65 gene sequencing: one species identified as M. chelonae by 

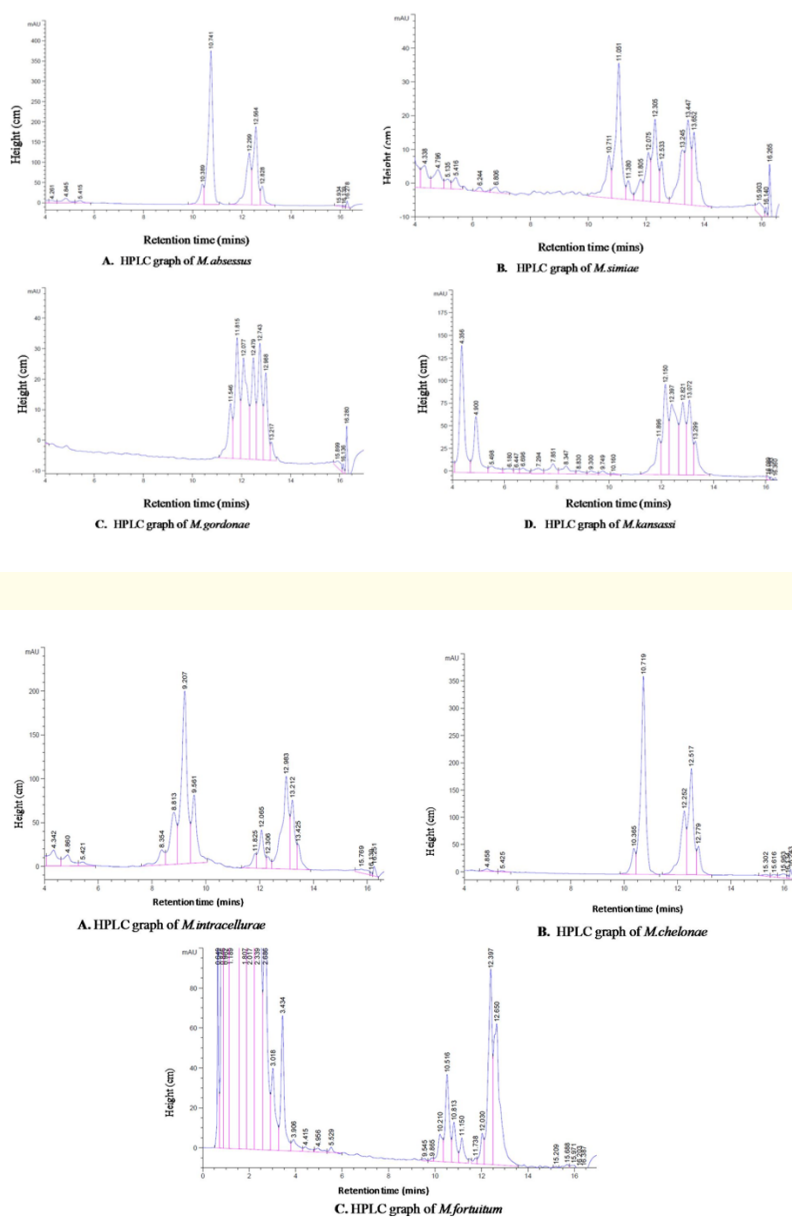

Figure 4

a: HPLC graphs of NTM Species.

b: HPLC graphs of NTM Species.

LPA and HPLC was identified as M. abscessus by both ITS and hsp65 gene sequencing. Interestingly, one isolate that could be identified neither by LPA nor by HPLC was identified using ITS and $h s p 65$ gene sequencing as Nocardia cyriacigeorgica (Table 1) and all $M$. abscessus were identified as subsp. Bolletii.

\section{Discussion}

Screening for Mycobacterium tuberculosis (MTB) using smear microscopy in patients presenting with a history suggestive of PTB is the cornerstone of tuberculosis control strategy, especially in high-burden, resource-limited countries like India. Even newer PCR-based molecular diagnostic tests such as GeneXpert MTB/RIF cannot identify NTM species, whose isolation depends on the cul-

\begin{tabular}{|c|c|c|c|}
\hline $\begin{array}{l}\text { S. } \\
\text { No. }\end{array}$ & $\begin{array}{c}\text { GenoType }^{\mathrm{R}} \\
\text { Mycobacterium } \\
\text { CM/AS kit (LPA) }\end{array}$ & $\begin{array}{l}\text { HPLC through } \\
\text { mycolic acid } \\
\text { analysis }\end{array}$ & $\begin{array}{c}\text { ITS region } \\
\text { sequencing by } \\
\text { automated DNA } \\
\text { sequencer }\end{array}$ \\
\hline 1. & M. intracellulare & M. intracellulare & M. intracellulare \\
\hline 2. & M. abscessus & M. abscessus & M. abscessus \\
\hline 3. & M. abscessus & M. abscessus & M. abscessus \\
\hline 4. & GC neg & Unidentified & $\begin{array}{l}\text { Nocardia cyriacigeor } \\
\text { gica }\end{array}$ \\
\hline 5. & M. abscessus & M. abscessus & M. abscessus \\
\hline 6. & M. gordonae & M. gordonae & M. gordonae \\
\hline 7. & M. intracellulare & M. intracellulare & M. intracellulare \\
\hline 8. & Invalid & M. kansasii & M. kansasii \\
\hline 9. & M. chelonae & M. chelonae & M. abscessus \\
\hline 10. & M. gordonae & M. gordonae & M. gordonae \\
\hline 11. & M. simiae & M. simiae & M. simiae \\
\hline 12. & M. abscessus & M. abscessus & M. abscessus \\
\hline 13. & M. intracellulare & M. intracellulare & M. intracellulare \\
\hline 14. & M. intracellulare & M. intracellulare & M. intracellulare \\
\hline 15. & M. intracellulare & M. intracellulare & M. intracellulare \\
\hline 16. & M. intracellulare & M. intracellulare & M. intracellulare \\
\hline 17. & M. intracellulare & M. intracellulare & M. intracellulare \\
\hline 18. & M. intracellulare & M. intracellulare & M. intracellulare \\
\hline 19. & M. intracellulare & M. intracellulare & M. intracellulare \\
\hline 20. & M. abscessus & M. abscessus & M. abscessus \\
\hline 21. & M. gordonae & M. gordonae & M. gordonae \\
\hline
\end{tabular}

Table 1: Comparison of LPA, HPLC and ITS region of ribosomal gene sequencing methods for isolation and species identification in 21 ICA negative culture isolates grown in liquid culture (MGIT960).

Definition of abbreviations: GC neg: Genus Control Negative; Noise: Unintrepertable Graph by Instruments; CM/AS: Common Mycobacteria/Additional Species of Mycobacteria; ICA: Immunochromatographic Assay: LPA: Line Probe Assay; HPLC: High Performance Liquid Chromatography; AFB: Acid-fast Bacilli; $M t b$, Mycobacterium tuberculosis NTM: Non-tuberculous Mycobacteria; ITS: Internal Transcribed Spacer; NCBI: National Centre for Biotechnology Information.

ture and other specialized techniques. Moreover, the techniques are available only at selected centres. All these factors highlight the importance of high clinical suspicion and timely referral of patients with NTM infections for appropriate testing. NTM infections 
present a unique challenge to clinicians, as their clinical and radiological presentations are similar to those of MTB, and because they show acid-fast reaction to ZN staining, which is usually the only test being performed at peripheral health centres before starting antituberculosis therapy [13]. This leads to inappropriate treatment of NTM cases, sometimes even with MDR-TB regimens in view of lack of response to conventional anti-TB drugs. Since a culture-positive result for NTM is an absolute diagnostic criterion according to ATS guidelines and because treatment regimens differ according to the species, rapid species identification of NTM is essential for appropriate treatment $[14,15]$.

The data on various techniques for NTM isolation from India are limited, with no study reporting ITS sequencing results so far. The present study was thus aimed at isolating and identifying NTM among persistently smear, culture-positive and ICA-negative isolates, where the patients infected were not responding to conventional anti-TB drugs. We used HPLC, LPA, and ITS and hsp65 gene sequencing and compared the results of the four methods to identify the best method for NTM species identification. Our study provides pivotal information on the potentially pathogenic range of prevailing NTM species among HIV-negative sputum-positive cases from a tertiary care centre in India.

Most pathogenic NTM species are resistant to first-line anti-TB drugs and cause disease progression with persistent and often irreversible loss of lung function [2]. A vast majority of NTM cases come to light when the patient is investigated for suspected drugresistant tuberculosis. A Chinese study revealed that about $30 \%$ of NTM isolates were from suspected MDR-TB cases, which corroborates the findings of our study [14]. Prevalence of NTM isolates can be quite high in EPTB cases, as demonstrated by a recent study from New Delhi, India, in which $12.4 \%$ of EPTB cases were actually NTM infections. The same study also identified NTM isolates in $17.4 \%$ of PTB cases who were on MDR-TB regimen [12].

The Canters for Disease Control and Prevention (CDC) proposes the use of HPLC to aid in the differentiation between tubercular and non-tubercular Mycobacteria [16]. In our study, of the 21 suspected NTM isolates, HPLC correctly identified 20 isolates as NTM and only one isolate was not identified; therefore, in resource-limited settings, HPLC can be a good choice for species identification. However, the turnover time (TOT) is relatively high and the requiresspecial training to perform this test are the major limitations forthe use of this modality [4].

The NTM isolates in our study were also tested using the GenoType ${ }^{\circledR}$ CM kit based on the principle of probe hybridization. Nine- teen of the 21 NTM isolates were identified using the GenoType ${ }^{\circledR}$ CM kit with one invalid result. Two unidentified isolates were then tested using the GenoType ${ }^{\circledR}$ AS kit, which identified one isolate as M. simiae, and the other isolate was found to not be a NTM. Another study from Lucknow, India, on pulmonary specimens evaluated the performance of GenoType ${ }^{\circledR}$ Mycobacterium CM/AS and reported a sensitivity of $99.3 \%$ and specificity of $98.3 \%$ for NTM species identification [17]. The most common NTM species reported in the study from Lucknow was Mycobacterium fortuitum (20, 33.3\%) among rapid growing Mycobacteria, and M. intracellulare (11, 18.3\%) among the slow-growing Mycobacteria [16]. Another study from Mumbai, India showed that M. abscessus (59\%) and M. fortuitum (41\%) were the dominant species [18]. In the present study, M. intracellulare (43\%) was found to be the predominant species, followed by M. gordonae (24\%) and M. abscessus (14\%).

Another study from Australia reported concordant results between LPA and HPLC for NTM identification among 119/131 clinical isolates [19]. These results were further substantiated in the present study, which showed $100 \%$ concordance between the two techniques.

One isolate that could not be detected by either LPA or HPLC was later identified as $N$. cyriacigeorgica by ITS sequencing. Nocardia infection is important and can silently present as pulmonary and extra- pulmonary diseases indistinguishable from Mycobacterium tuberculosis infections. $N$. cyriacigeorgica has been isolated predominantly from respiratory samples, although isolation from other sites is not infrequent and has been reported from Europe, Asia, and North America [20]. Being acid-fast, this species is often missed, and patients are subjected to erroneous treatment, which may contribute to significant morbidity and mortality. A discordant result between ITS sequencing and LPA/HPLC was found for one species, which was identified incorrectly as M. chelonae using HPLC and LPA and was later identified correctly as M. abscessus using ITS sequencing. This difference in the results obtained using the different methods could be explained by the complicated taxonomical relationship between M. chelonae and M. abscessus, both of which were earlier considered part of the M. chelonae-abscessus group. It was only later that $M$. chelonae was characterized as a separate species; however, clarity on this topic is still lacking in published literature [21,22].

International literature on the use of the ITS region for ribosomal gene sequencing for NTM species identification is very limited. To the best of our knowledge, this is the first study from Delhi, In- 
dia, to present such data and to report concordant results between the three techniques for characterization of NTM species.

One major limitation of this study was the lack of data on co-infection of MTB and NTM, as the tests were not performed on mixed cultures. This aspect has also been infrequently reported in previous studies and could be a relevant research question for future studies [23]. Nevertheless, the findings from the present study indicate that the use of ITS (16S-23S rDNA ITS sequences) sequencing is a rapid and reliable way of NTM species identification [24].

\section{Conclusion}

The rapidityof ITS along with hsp65 genes sequencing may help in the early and accurate identification of NTM species and subspecies and will aid in initiation of appropriate treatment regimen. The rapidity, cost-effectiveness, and reliabilityof this technique indicates that it has potential for use as a standard of careon a larger scale.

\section{Funding Support}

No funding to declare.

\section{Acknowledgments}

The authors are thankful to the AIIMS administration for providing appropriate infrastructure; Central TB Division, Ministry of Health and Family Welfare, Govt. of India; State TB cell, Foundation of Innovative New Diagnostics (FIND), India for logistic support. Authors acknowledge the help of the staff at IRL, AIIMS for sample collection and processing.

\section{Bibliography}

1. Tortoli E. "Impact of genotypic studies on mycobacterial taxonomy: the new mycobacteria of the 1990s". Clinical Microbiology Review 16 (2003): 319-354.

2. Heifets L. "Mycobacterial infections caused by non tuberculous mycobacteria". Seminars in Respiratory and Critical Care Medicine 25 (2004): 283-295.

3. Kadam M., et al. "Can cord formation in Bactec MGIT-960 medium be used as a presumptive method for identification of $\mathrm{M}$. tuberculosis complex". Indian Journal of Tuberculosis (2010): 234-453.

4. Jeong J., et al. "The Use of High Performance Liquid Chromatography to Speciate and Characterize the Epidemiology of Mycobacteria”. Laboratory Medicne 42 (2011): 10.
5. Park H., et al. "Detection and Identification of Mycobacteria by Amplification of the Internal Transcribed Spacer Regions with Genus and Species-Specific PCR Primers". Journal of Clinical Microbiology (2000): 4080-4085.

6. Telenti A., et al. "Rapid identification of mycobacteria to the species level by polymerase chain reaction and restriction enzyme analysis". Journal of Clinical Microbiology 31 (1993): 175-178.

7. de Zwaan R., et al. "Utility of rpoB gene sequencing for identification of nontuberculous mycobacteria in the Netherlands". Journal of Clinical Microbiology 52.7 (2014): 544-551.

8. Kim BJ., et al. "Mycobacterium paragordonae sp. nov., a slowly growing, scotochromogenic species closely related to Mycobacterium gordonae". International Journal of Systematic and Evolutionary Microbiology 64.1 (2014): 39-45.

9. Kubica GP., et al. "Sputum digestion and decontamination with Nacetyl-L-cysteine-sodium hydroxide for culture of Mycobacteria”. American Review of Respiratory Disease Returns 87 (1963): 775-779.

10. Central TB. "Division Ministry of Health and Family Welfare. Training Manual for Mycobacterium tuberculosis Culture and Drug susceptibility testing". New Delhi, India (2009).

11. Runyon E. "Typical mycobacteria: their classification". American Review of Respiratory Disease 91 (1965): 288-289.

12. Sharma R., et al. "A case of non-healing herniorrhaphy: common problem, uncommon cause". International Journal of Medical Laboratory Research 2.3 (2017): 63-66.

13. Gopinath K and Singh S. "Non-tuberculous Mycobacteria in TB endemic countries: are we neglecting the danger?" PLOS Neglected Tropical Diseases 4 (2010): e615.

14. Jing H., et al. "Prevalence of nontuberculous mycobacteria infection, China, 2004-2009". Emerging Infectious Diseases 18 (2012): 527-528.

15. Griffith DE., et al. "An official ATS/IDSA statement: diagnosis, treatment, and prevention of nontuberculous mycobacterial diseases". American Journal of Respiratory and Critical Care Medicine 175 (2007): 367-416.

16. CDC. "Standardized Method for HPLC Identification of Mycobacteria". Atlanta, GA: Centers for Disease Control and Prevention (1996). 
17. Singh AK., et al. "Role of GenoType® Mycobacterium Common Mycobacteria/ Additional Species Assay for Rapid Differentiation between Mycobacterium tuberculosis Complex and Different Species of Nontuberculous Mycobacteria". Journal of Laboratory Physician 5 (2013): 2.

18. Shenai S., et al. "Time to identify and define non-tuberculous mycobacteria in a tuberculosis-endemic region". International Journal of Tuberculosis and Lung Disease 14 (2010): 10011008.

19. Lee AS., et al. "Identification of non-tuberculous mycobacteria: utility of the GenoType Mycobacterium CM/AS assay compared with HPLC and 16S rRNA gene sequencing". Journal of Medical Microbiology 58 (2009): 900-904.

20. Cargill JS., et al. "Nocardiacyriacigeorgica: a case of endocarditis with disseminated soft-tissue infection". Journal of Medical Microbiology 59 (2010): 224-230.

21. Kusunoki S and Ezaki T. "Proposal of Mycobacterium Peregrinum sp.nov., nom.rev., and elevation of Mycobacterium Chelonaesubsp. abscessus to species status: M. abscessuscomb. nov". International Journal of Systematic and Evolutionary Microbiology 42.2 (1992): 240-245.

22. Simmon KE., et al. "Mycobacterium chelonae-abscessus complex associated with sinopulmonary disease, North-eastern USA". Emerging Infectious Diseases 17.9 (2011): 1692-700.

23. Maiga M., et al. "Failure to recognize nontuberculous Mycobacteria leads to misdiagnosis of chronic pulmonary tuberculosis". PLoS ONE 7 (2012): e36902.

24. Roth A., et al. "Differentiation of phylogenetically related slowly growing mycobacteria based on 16S-23S rRNA gene internal transcribed spacer sequences". Journal of Clinical Microbiology 36 (1998): 139-147.

\section{Volume 5 Issue 9 September 2021}

(C) All rights are reserved by Pankaj Jorwal., et al. 